\title{
The Expanding Role of Stress Echocardio- graphy in Hypertrophic Cardiomyopathy
}

\author{
Eszter Dalma Pálinkás', Quirino Ciampi², Eugenio Picano³
}

\author{
${ }^{1}$ Albert Szent-Gyorgy University Medical School, Szeged, Hungary \\ ${ }^{2}$ Cardiology Division, Fatebenefratelli Hospital, Benevento, Italy \\ ${ }^{3} \mathrm{CNR}$ Institute of Clinical Physiology, Pisa, Italy
}

\author{
Address for correspondence: \\ Eugenio Picano, MD, PhD \\ Consiglio Nazionale Ricerche - Istituto Fisiologia Clinica \\ CNR Research Campus - Via Moruzzi, 1-Building C, room 130 \\ 56124 Pisa - Italy \\ E-mail: picano@ifc.cnr.it
}

In the European Society of Cardiology 2014 guidelines on hypertrophic cardiomyopathy (HCM), exercise stress echo (SE) is recommended in symptomatic patients if bedside manoeuvres fail to induce left ventricular outflow tract gradient (LVOTG) $\geq 50 \mathrm{mmHg}$ and is rated as class I, level of evidence B. The recommendation is class II b, level of evidence C, in asymptomatic patients. This evidence-based approach uses one stress (exercise), one parameter (LVOTG), and one target (obstruction) in one HCM patient (symptomatic without obstruction at rest). However, the omnivorous versatility of contemporary SE appears ideally suited to unmask the functional heterogeneity underlying the similar morphology and clinical presentation of HCM. At least 7 parameters converge conceptually, logistically, and methodologically in the ABCDEFG-SE protocol in HCM. They are: 1) regional wall motion abnormalities (step A); 2) B-lines by lung ultrasound (step B), possibly with other indices of diastolic function such as E/e' and systolic pulmonary arterial pressure (from tricuspid regurgitant jet velocity or acceleration time of forward systolic pulmonary flow); 3) left ventricular contractile and preload reserve (step C) based respectively on force (systolic arterial pressure by cuff sphygmomanometer/endsystolic volume from 2-D) and end-diastolic volume; 4) coronary flow velocity reserve (step D) in left anterior descending coronary artery (with pulsed wave-Doppler); 5) heart rate reserve (peak/rest heart rate) from EKG (step E); 6) mitral valve regurgitate flow (step F); and 7) LVOTG (step G). ABCDEFG-SE allows a comprehensive assessment of inducible ischemia $(A)$, lung water $(B)$, myocardial systolic and diastolic function (C), coronary microcirculation (D), autonomic function $(E)$, mitral regurgitation (F) and intraventricular obstruction (G). Yesterday SE used LVOTG for HCM with a one-fits-all approach. SE Today, SE may exploit its unsurpassed versatility to unmask different pathophysiological mechanisms and potential therapeutic targets in a personalized and comprehensive approach to HCM.

Keywords: intraventricular gradient, hypertrophic cardiomyopathy, ischemia, stress echo

\section{List of abbreviations}

CAD: coronary artery disease; ESC: European Society of Cardiology; HCM: hypertrophic cardiomyopathy; HRR: heart rate reserve; LV: left ventricle; LVOTO: left ventricular outflow tract obstruction; RWMA: regional wall motion abnormalities; SAM: systolic anterior motion; SE: stress echocardiography 


\section{Introduction}

Hypertrophic cardiomyopathy $(\mathrm{HCM})$ is the most common monogenic disease of the myocardium, with 1:500 prevalence in the general population worldwide, although often misdiagnosed or neglected. HCM is defined by the presence of increased left ventricular (LV) wall thickness $\geq 15 \mathrm{~mm}$ by any imaging modality in one or more LV myocardial segments that is not solely explained by abnormal loading conditions, and occurs in the absence of other detectable causes (1). A lower threshold for LV thickness (i.e.: $\geq 13 \mathrm{~mm}$ ) is adopted in first-degree relatives of patients with unequivocal disease. Clinical diagnosis is customarily made with two-dimensional echocardiography by detection of increased LV wall thickness, usually in the presence of a small LV cavity, after suspicion has been raised by the clinical profile or as a part of screening. In most patients, HCM is caused by mutations in genes encoding contractile proteins of the cardiac sarcomere, Z-disk and intracellular calcium handling pathways. In over one-third of patients, however, the genetic basis of the disease remains unresolved (2).

$\mathrm{HCM}$ is often characterized by a stable and uneventful clinical course, and may be diagnosed late in life. However, about $50 \%$ of patients experience symptoms, and $15 \%$ progress towards LV dysfunction and heart failure, including 5\% ultimately developing end-stage disease. In addition, the condition is associated with a $0.5-1 \%$ annual risk of sudden cardiac death (2). Prior history of cardiac arrest is an obvious indicator of risk representing a clearcut indication for the implantable cardioverter defibrillator (2). In primary prevention, however, arrhythmic risk prediction proves extremely challenging, due to the low event rate and low positive predictive accuracy of risk factors identified to date. Current recommendations of the European Society of Cardiology (ESC) include individual multiparametric assessment of annual sudden death risk score comprising age, family history of sudden death, personal history of unexplained syncope, left atrial diameter, maximal wall thickness, and LV outflow gradients (at rest or during Valsalva) with baseline transthoracic echocardiography (1). Resting echocardiography with serial evaluations over time provides additional markers of high risk such as progressive wall thinning, declining systolic function, the presence of LV apical aneurysms, severe mitral insufficiency and restrictive LV filling pattern (3). In all patients undergoing septal alcohol ablation, intracoronary contrast echocardiography is recommended to ensure correct localization of alcohol (1).

\section{Stress echo in guidelines and recommendations}

For decades, exercise was considered a strict contraindication to exercise testing, for the fear of hemodynamic catastrophes. It is now established that a resting evaluation cannot adequately represent the dynamic changes occurring during exercise in $\mathrm{HCM}$ patients, whose functional features are very heterogeneous from patient to patient (4), may vary in the same patient from rest to stress, and are strikingly affected by therapeutic interventions (5). Therefore, according to recent 2017 joint recommendations of European Association of Cardiovascular Imaging-American Society of Echocardiography societies on stress echo beyond coronary artery disease, "exercise SE is safe and commonly used especially in patients with equivocal symptoms, to determine functional capacity prior to a corrective therapeutic procedure, and for individual risk stratification". In the ESC 2014 guidelines on HCM, exercise SE is recommended in symptomatic patients if bedside manoeuvres fail to induce LVOTO $\geq 50 \mathrm{mmHg}$ and is rated as class lla ("should be considered"), level of evidence B ("data derived from a single randomized clinical trial or large non-randomized studies"). The recommendation is class II b ("may be considered"), level of evidence C ("consensus of opinion of the experts and/or small studies, retrospective studies, registries"), in asymptomatic patients. Other useful information beyond the LVOTO can be obtained during SE, since - according to 2017 recommendations - "abnormal blood pressure response to exercise, blunted systolic and diastolic reserve, and worsened MR are associated with poor exercise capacity and outcome. SE is not indicated when a gradient $>50 \mathrm{mmHg}$ is present at rest". The recommended stress is exercise with standing, sitting or semi-supine position. Gradients are higher in the post-exercise standing position or in post-prandial exercise, in physiological conditions when preload decreases. All these information's represent a substantial contribution to clinical management and risk stratification (6). According to an expert consensus of European Association of Cardiovascular Imaging in 2015, "SE can be used for functional imaging in HCM with coronary flow reserve assessment on the left anterior descending artery with concomitant wall motion analysis which provides diagnostic and prognostic information and may allow the distinction between obstructive epicardial coronary disease and microvascular ischemia". In fact, microvascular angina is identified by the combination of normal regional wall motion and blunted coronary flow reserve, while obstructive epicardial CAD shows a reduced flow reserve associated with RWMA (3). According to 2017 recommendations, vasodilation is the preferred modality for the evaluation of coronary flow velocity reserve (7).

\section{Stress echo beyond guidelines: the ABCDEFG protocol}

In analogy with the standard state-of-the-art protocol $A B C D E$ used and disseminated in the Stress echo 
TABLE 1. Clinically relevant information derived from stress echocardiography in HCM

\begin{tabular}{|l|l|l|l|}
\hline Step & Target & Evidence level & $\begin{array}{l}\text { Possible examples of Management } \\
\text { implications }\end{array}$ \\
\hline $\begin{array}{l}\text { A-wall motion } \\
\text { abnormality }\end{array}$ & Myocardial ischemia & Guidelines & $\begin{array}{l}\text { Revascularization when CAD present with angina/ } \\
\text { dyspnea }\end{array}$ \\
\hline B: B-lines & Pulmonary congestion & Preliminary data & Decongestion therapy in dyspnea? \\
\hline C: Contractile reserve & Left ventricular function & Preliminary data & Molecular anti-myosin therapy when increased? \\
\hline D: Doppler & Coronary microcirculation & Expert consensus & Cholesterol lowering drugs at LDL-C <55 mg/dL? \\
\hline E: EKG-based HRR & Cardiac autonomic function & Preliminary data & Rate responsive atrial pacing? \\
\hline F: Flow of MR & Mitral valve dysfunction & Expert consensus & MV replacement when myectomy not enough? \\
\hline G: Gradient in LVOTO & LV obstruction & Guidelines & $\begin{array}{l}\text { Beta-blockers in symptomatic, myectomy/myotomy } \\
\text { in severely symptomatic refractory to medical therapy }\end{array}$ \\
\hline
\end{tabular}

2020 study for the comprehensive functional testing of patients with suspected or known CAD and/or heart failure $(7,8,9)$, the most comprehensive protocol adapted to the complexity and heterogeneity of HCM patients can be schematized as the ABCDEFG protocol. Each step of the stress echo evaluation corresponds to a different pathophysiological target in HCM (Table 1). The assessment is ideally performed during exercise, but at least for some parameters the same evaluation (for instance, coronary flow velocity reserve with vasodilator stress) can be best performed during pharmacological testing when exercise is not feasible or has contraindications or simply too technically demanding. In theory, each parameter has a different pathophysiological target and therefore a different therapeutic counterpart. We currently miss the supportive evidence necessary to adopt a tailored SE-guided approach, but the existing data already challenge the one-fits all approach which does not incorporate in decision-making the tremendous functional heterogeneity of the disease also mirrored by the heterogeneity of functional responses during SE.

\section{A for regional wall motion abnormalities}

Chest pain is a frequent symptom in $\mathrm{HCM}$, and angiographically assessed coronary artery disease (CAD) is found in about $20 \%$ of patients and is associated with an increased mortality. Many HCM patients with chest pain do not show epicardial disease, and their symptoms are likely due to microvascular disease. Perfusion changes can be present during stress scintigraphy and are linked to microvascular disease, with a poor specificity for the detection of underlying epicardial CAD. For the purposes of noninvasive identification of concomitant CAD, regional wall motion abnormalities (RWMA) in HCM patients are substantially more specific than perfusion abnormalities and ST-segment depression, but also may suffer from false positive responses especially in presence of marked hypertrophy $(10,11)$. Therefore, a negative test for RWMA is useful for excluding functionally significant CAD, or, in younger patients, tunneling of the left anterior descending coronary artery, but a positive test is less useful for including a CAD and indicating an ischemia-driven revascularization. In patients with chest pain and positive SE for inducible RWMA, a noninvasive coronary computed angiography is indicated prior to referring the patient to ischemia-driven revascularization. RWMA occur in $6 \%$ of HCM patients and are a strong predictor of adverse outcome even in absence of underlying coronary artery disease $(12,13,14)$.

\section{B for B-lines}

B-lines (also known as ultrasound lung comets) are a pre-clinical and pre-radiological sign of pulmonary congestion assessed with lung ultrasound (15). Their appearance during stress indicates an acute backward heart failure which may have different origins, from excessive afterload mismatch to diastolic dysfunction, acute functional mitral insufficiency, inducible ischemia and RWMA (16). All these factors can come into play during stress in HCM patients. Diastolic reserve is usually assessed during SE with E/e' and systolic pulmonary arterial pressure from tricuspid regurgitant jet, both considerably less feasible than B-lines during stress. When tricuspid regurgitant jet is absent, the acceleration time of the physiologic systolic pulmonary flow is a reasonable alternative. With increasing pulmonary pressures, acceleration time shortens when tricuspid jet velocity rises (17). B-lines are much easier to obtain and to analyze, and are also feasible in all patients in $<1$ minute with the simplified 4-site scan (16). Conceptualyly, they focus on pulmonary congestion, differently from E/e' measuring LV filling and systolic pulmonary arterial pressure assessing systemic hemodynamic congestion. The appearance of B-lines is linearly, closely and negatively correlated with maximal oxygen consumption on cardiopulmonary testing in heart failure patients (18) and may usefully integrate the prognostic profiling of the patient, possibly indicating a personalized SEguided approach to lung decongestion therapy. Diuretics are generally considered contraindicated in $\mathrm{HCM}$ since they may induce dehydration which may induce 
worsening of obstruction. It is however accepted that they can be used in HCM patients with dyspnea and pulmonary congestion (19). The observation of B-lines might eventually drive a more personalized use of diuretics in selected patients showing moderate to severe pulmonary congestion at rest or during stress.

\section{C for contractile reserve}

Contractile function is especially important in HCM patients, and is better defined with load-independent force (which also incorporates the value of LVOTG at rest and peak stress) rather than with ejection fraction $(20,21)$. At initial stages of HCM, the peculiar pattern is a normal-increased value of baseline ejection fraction, with a blunted increase of force during stress, associated with downregulation of SERCA-2 mRNA, resulting in altered calcium handling which may contribute to reduced contractile reserve (22). About 1 of 4 to 5 uncomplicated HCM patients have a defect in LV force reserve in spite of normal values of rest and stress ejection fraction, and this defect is associated to a higher incidence of adverse events (23). The identification of contractile state of the LV in HCM is especially relevant since in some mutations the earliest sign of the disease is a hyperdynamic contraction. For instance, the two most frequently mutated $\mathrm{HCM}$ genes encode $\beta$-cardiac myosin heavy chain (MYH7) and myosin-binding protein C (MYBPC3).Young carriers of either MYH7 or MYBPC3 mutation have shown hyperdynamic contraction and impaired relaxation that precede the appearance of myocardial hypertrophy. A small molecule, MYK461 , reduces contractility by decreasing the adenosine triphosphatase activity of the myosin heavy chain. The early, chronic administration of MYK-461 suppresses the development of left ventricular hypertrophy, cardiomyocyte disarray, and myocardial fibrosis indicating that hyperdynamic contraction is essential for HCM pathobiology (24). The possibility of incorporating a simple and reproducible biomarker of LV contractility such as force in the SE assessment of HCM seems especially attractive as new molecular therapies of contractility are now being tested in clinical studies.

\section{D for Doppler-based coronary flow velocity reserve}

Symptoms and signs of myocardial ischemia are often found in patients with $\mathrm{HCM}$, in the presence of angiographically normal coronary arteries, reflecting microvascular disease exacerbated by myocardial hypertrophy and dynamic LV obstruction (1, 2). Myocardial ischemia is primarily due to extensive remodeling of the intramural coronary arterioles and blunted coronary artery reserve, occurring not only in the hypertrophied septum, but also in the less hypertrophied left ventricular free wall. Microvascular ischemia is believed to contribute to the most severe manifestations of HCM including ventricular arrhythmias, sudden death and progressive left ventricular remodeling, and the degree of microvascular dysfunction is an independent predictor of long-term deterioration and death from cardiovascular causes. Coronary flow velocity reserve is reduced in about one-third of HCM patients, as first described with accurate but costly positron emission tomography imaging methods (25). The measurement is equally reliable and substantially simpler with Doppler echocardiography on mid-distal left anterior descending artery with vasodilators. The feasibility is $>95 \%$ in general population (9), and higher in HCM since the thick septum, the large coronary diameter and the increased resting flow make the detection and sampling of coronary flow faster and easier. As in other models of microvascular disease, such as cardiac syndrome $\mathrm{X}$ or arterial hypertension $(26,27)$, simple ST-segment changes are frequently elicited during vasodilator stress in $\mathrm{HCM}$ patients, representing reduced flow reserve and true subendocardial underperfusion (28) and are associated with worse outcome $(29,30)$. Of note, the absence of RWMA does not necessarily contradict the ischemic nature of chest pain and ST-segment depression in HCM patients. Rather, because of the largely subendocardial nature of ischemia, as well as the considerable LV wall thickness, the degrees of transmural involvement are less likely to reach the critical mass of ischemic tissue needed to determine wall motion and thickening abnormalities. The presence and degree of impairment of CFVR is largely unrelated to the presence and degree of left ventricular hypertrophy, although more pronounced in presence of LVOTO and relieved by myectomy (31). The reduction of CFVR shows a striking prognostic value, clearly superior to LVOTO $(32,33)$.

The presence of a dominant phenotype with coronary microvessel dysfunction could suggest a therapy with beta-blockers and calcium-antagonists, an aggressive therapy of risk factors toxic for microcirculation such as glycemia (with insulin) or LDL-cholesterol (with statin or PCSK9-inhibitors), or possibly a selective therapeutic intervention with drugs acting on coronary microcirculation (34) and known to improve coronary flow reserve in $\mathrm{HCM}(35)$.

\section{E for EKG-based heart rate reserve}

Even in the stress imaging era, non-imaging information simply based on old-fashioned EKG and cuff sphygmomanometer can supply surprising pathophysiological and prognostic information and should be incorporated in the analysis and reporting of stress response. The 3 main categories of information, additive and complementary to imaging, relate to systolic blood pressure response, inducible arrhythmias and heart rate response.

Abnormal blood pressure response to exercise is defined as exercise-induced hypotension (any decrease in systolic blood pressure below baseline in the absence of an initial rise with exercise, or a sustained decrease 
of $>20 \mathrm{mmHg}$ during exercise following an initial rise) or failure to increase blood pressure (a systolic blood pressure rise of less than $20 \mathrm{mmHg}$ from baseline) (1). Abnormal blood pressure response to exercise is observed up to one-quarter of HCM patients, and is believed to identify hemodynamic instability secondary to LVOTO, diastolic dysfunction, microvascular ischemia and inappropriate peripheral vasodilatation, representing an independent predictor of sudden cardiac death (2). Whether therapeutic interventions such as surgical myectomy, alcohol septal ablation or pharmacological therapy may consistently resolve abnormal blood pressure response, and whether this in turn may translate into a survival benefit, is as yet unresolved. Exercise-induced hypotension is an indication for interruption of stress testing, and may require positioning the patient in the supine or Trendelemburg position when symptomatic.

Exercise induced ventricular arrhythmias are uncommon in HCM patients but, when present, predict major arrhythmic events. The clinical relevance of ventricular arrhythmias in HCM is inversely related to age, and should be considered with particular suspicion in the pediatric/adolescent age group $(1,2)$.

Heart rate reserve (HRR) is easily measured from 1 lead EKG (present by protocol in the echo monitor) as the peak/rest heart rate ratio. A blunted $\operatorname{HRR}(<1.80)$ is a marker of cardiac autonomic dysfunction and reduced sympathetic reserve (36). Impairment of HRR is associated with increased risk of cardiovascular outcomes and all-cause mortality in the general population, with independent value over inducible RWMA during SE with exercise (36), dobutamine (37) and dipyridamole (8). In HCM, a reduced HRR is associated with a lower peak oxygen consumption at cardiopulmonary testing, higher degree of myocardial fibrosis with delayed enhancement with cardiovascular magnetic resonance and worse outcome (38). At least in theory, chronotropic incompetence of HCM might be selectively corrected with atrial pacing.

\section{$F$ for functional mitral regurgitation}

LV outflow obstruction is a pathophysiological conspiracy caused by the concomitance of marked mitral leaflet elongation, hyper-contractile small or normal-sized LV, abnormally positioned papillary muscles, small LV outflow tract dimensions and abnormally directed anterograde flow in systole due to septal hypertrophy, leading to systolic anterior motion (SAM) of the mitral valve. SAM is in turn associated with variable degrees of functional (SAM-related) mitral regurgitation, due to loss of leaflet coaptation, which is mid-to-late systolic and infero-laterally oriented (1). In the elderly, posterior mitral annulus calcification, anteroposition of the mitral apparatus and sigmoid septal morphology with decreased septal-aortic angle increase the likelihood of SAM and obstruction, which may develop in previously unobstructed patients. Due to its dynamic nature, the severity of mitral regurgitation varies with the degree of LVOTO, may increase significantly during effort, and an exercise-induce at least moderate mitral regurgitation represents a main determinant of symptoms and prognosis (39). In many cases, surgical myectomy (or alcohol septal ablation) may be sufficient to correct MR, which decreases with the reduced obstruction. However, since it can be also primarily due to morphologic changes such as elongation of mitral leaflets or papillary muscle abnormalities, in specific instances, concomitant MV repair or novel percutaneous interventions may be required (1).

\section{G for intraventricular pressure Gradient} Intraventricular obstruction is an additional mechanism of ischemia, determining a disproportionate increase in oxygen demand and reduction in subendocardial flow supply due to increased extravascular resistances (41). Most HCM patients have the propensity to develop intraventricular gradients under resting or physiologically provocable conditions. Such dynamic obstruction generally occurs at the LV outflow, produced by SAM of the mitral valve causing ventricular septal contact.

Dynamic obstruction may occur at different sites besides the LVOT, including the LV mid-ventricle (due to interposition and septal contact of the anterior papillary muscle) and the RVOT (due to a sphincter-like mechanism occurring at the level of the crista supraventricularis) (42). Fixed, anatomic obstruction due to sub-aortic membranes should be carefully excluded. Each of these mechanism should be systemically assessed, since management strategies may differ, particularly when interventions aimed at relieving LVOTO are considered $(1,2)$. Dynamic assessment of LVOTO (at rest, during bedside manoeuvers and exercise) holds important prognostic information and plays a pivotal role for the assessment of symptoms $(2,3)$.

By convention, LVOTO is defined as an instantaneous peak Doppler LV outflow tract pressure gradient $\geq 30$ $\mathrm{mmHg}$ at rest or during physiological provocation (i.e.: Valsalva manoeuver, standing and isotonic exercise). A gradient of $\geq 50 \mathrm{mmHg}$ is usually considered the threshold at which LVOTO becomes hemodynamically important and invasive treatment may be appropriate $(2,3)$. Up to one-third of patients have obstruction at rest (peak instantaneous gradient $>30 \mathrm{mmHg}$ ), another third have labile, physiologically provoked gradient $\quad<30$ $\mathrm{mmHg}$ at rest and physiologically provoked gradient $>30 \mathrm{mmHg}$ ) and only one-third have true non-obstructive forms (rest and physiologically provoked gradient $<30 \mathrm{mmHg}$ ) (41). Provokable gradients may characteristically peak after cessation of exercise, due to the effects of the ensuing fall in peripheral resistance.

Of note, while not all patients with HCM have LVOTO, it is also true that not all subjects with stress-induced gradients and LVOTO have HCM. Significant gradients can 
be observed during exercise or dobutamine in patients with Syndrome $X$ or hypertension, athletes, tako-tsubo syndrome, congenital heart diseases, following cardiac valve surgery, or in acute myocardial infarction, and can be precipitated by dehydration, reduction in preload and LV cavity size and/or increase in LV contractility.

\section{Technical issues and pitfalls}

In the vast majority of HCM patients, stress testing can be performed safely, including those with elevated LVOTO at rest who have no history of hemodynamic instability on effort. In fact, while HCM has long been considered a relative contraindication to stress testing, it is now well established that echocardiography during physiological exercise has minimal risk when performed in a controlled, supervised environment (6). Nevertheless, in a subset of $\mathrm{HCM}$ patients stress testing is not feasible or advisable, due inability to exercise, severe congestive symptoms (New York Heart Association class $\geq$ III), hemodynamic instability associated with LVOTO at rest, known effort-induced arrhythmias or severe comorbidities. Conversely, acoustic window quality is rarely a problem, given the young mean age of patients with $\mathrm{HCM}$.

Since many factors may affect LVOTO evaluation, a standardized approach is essential in order to have meaningful and comparable results across different laboratories. Different exercise modalities have been described in the literature, including semi-supine ergometer, treadmill (with image acquisition either du- ring exercise or at peak and supine immediately after exertion) upright bicycle, with imaging during exercise, at peak upright and immediately after exertion, upright and supine. The semi-supine exercise is a reasonable choice, although cardiac symptoms in these patients are noted most commonly when they are in the erect position, during or immediately after exertion (1). Substantial agreement between the magnitude of outflow gradients measured at the final step of the exercise test in the upright position and those gradients obtained in the supine position immediately following cessation of exercise has been reported, with post-exercise supine gradients overestimating somewhat the upright gradients on the average of $5 \mathrm{mmHg}$ for the study group (40). The timing of onset of obstruction was shown to dictate the degree to which exercise capacity was impaired in $\mathrm{HCM}$ patients with provokable gradients (40). The ESC guidelines suggest that "laboratories should develop and validate their own protocol and ensure that staff are properly trained in the procedure." In an ideal setting, care should be given to assessing degree of obstruction, mitral regurgitation, diastolic dysfunction, RWMA and pulmonary pressures at each step. Coronary flow velocity reserve can be assessed during exercise or in a separate same-day session with vasodilator stress. Whatever the stress, HRR should be reported. A frequent pitfall during effort involves the possibility of sampling MR instead of intraventricular gradients. Therefore, the utmost care should be employed to avoid this source of misinterpretation (6). B-lines by lung ultrasound are usually measured soon after the end of exercise, so not to interfere with other measurements (such as

\section{Risk stratification beyond LVOTG in HCM}

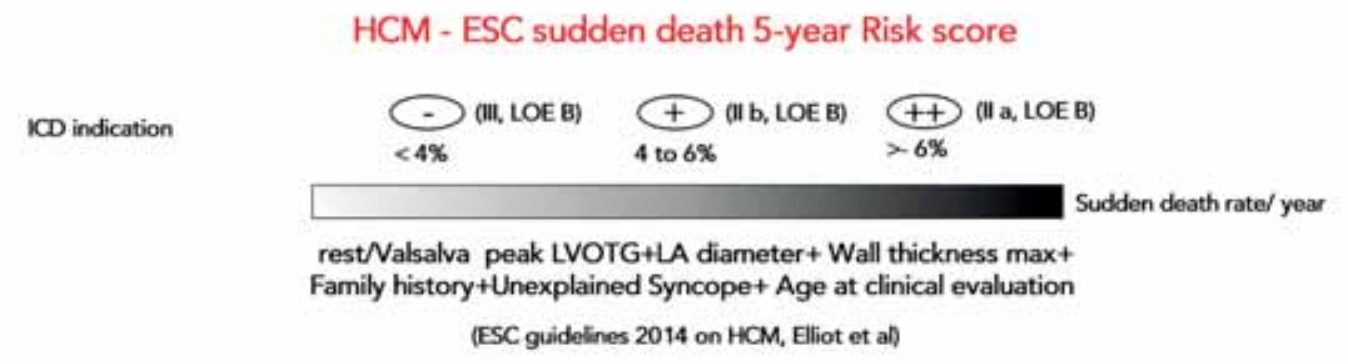

\begin{tabular}{ll}
\hline Nonischemic & Ischemic \\
Dry and Empty & Wet and Full \\
Strong and compliant & Weak and stiff \\
Warm & Cold \\
Fast & Slow \\
Non-severely regurgitant flows & Regurgitant \\
Non obstructive gradients & Obstructive
\end{tabular}

FIGURE 1. A working hypothesis: risk stratification with the ABCDEFG protocol. SE acts as a Newton's prism decoding the homogeneous white light of clinical presentation and resting transthoracic echocardiography findings in a spectrum of different responses and corresponding risks, when all parameters are normal (left side, green code, ABCDEFG negative) up to the extreme right when all parameters are abnormal (right side, red code, $A B C D E F G$ positive) 
LVOTO or mitral insufficiency of coronary flow velocity in left anterior descending artery) that instantaneously change at exercise interruption. B-lines remain visible for some minutes and therefore they can be acquired in the first minute after interruption of exercise without significant loss of information

\section{Conclusion}

HCM is a complex and heterogeneous disease with protean manifestations and the current unidimensional approach of SE mainly, if not exclusively, focused on LV obstruction does not exploit at the fullest the unique versatility of the technique. Its ubiquitous availability, low cost, radiation-free nature make SE ideally suited to be serially applied to HCM patients at all stages of disease and all ages of life to allow a comprehensive risk stratification in these complex patients (Figure 1). SE provides a cinematographic, not only snapshot, description of the development, evolution and progression of the disease from its preclinical pre-hypertrophic, to hypertrophic-stable, up to late stages of adverse remodeling and obvious resting LV dysfunction. This is particularly important today, since HCM is discovered early and HCM-related causes of death are only a minority in adult patients, with cancer and CAD being the leading cause after the age of 60 (41). It is especially important to avoid damage and risk from cumulative ionizing radiation exposure, which can be substantial in these patients if scintigraphy, positron emission tomography, coronary computed angiography and invasive angiography are used liberally (as done in the past) also at early age, leading to a substantial cumulative radiation exposure directly and linearly linked to cancer risk $(42,43)$. Another potential problem is the cumulative effects of gadolinium paramagnetic contrast agent, of special concern at young age and in patients repeating serial examinations over time as HCM patients often do especially for the evaluation of myocardial fibrosis, a known prognostic determinant (44). At least in principle, SE has the potential to discriminate, at low cost and without any known long-term risk, the various functional components, providing an unprecedented amount of information ideally suited to be combined with clinical and genetic data in the methodological framework of network medicine (45) and eventually paving the way to a personalized treatment driven by pathophysiology.

EDP is recipient of the Erasmus grant for medical students spent in Pisa in August 2019-April 2020

\section{The Authors have no conflict of interest to disclose}

\section{REFERENCES}

1. Elliott PM, Anastasakis A, Borger MA, et al. 2014 ESC Guidelines on diagnosis and management of hypertrophic cardiomyopathy: the Task Force for the Diagnosis and Management of Hypertrophic Cardiomyopathy of the European Society of Cardiology (ESC). Eur Heart J 2014; 35: 2733-2779. doi:10.1093/eurheartj/ehu284

2. Gersh BJ, Maron BJ, Bonow RO, et al 2011 ACCF/AHA Guideline for the Diagnosis and Treatment of Hypertrophic Cardiomyopathy. Circulation 2011; 124: e783-e831. doi: 10.1161/CIR.0b013e318223e2bd

3. Cardim N, Galderisi M, Edvardsen T, et al. Role of multimodality cardiac imaging in the management of patients with hypertrophic cardiomyopathy: an expert consensus of the European Association of Cardiovascular Imaging Endorsed by the Saudi Heart Association. Eur Heart J Cardiovasc Imaging 2015; 16: 280. doi: 10.1093/ ehjci/jeu291

4. Olivotto I, Nistri S, Picano E. Stress echocardiography in Hypertrophic Cardiomyopathy. In Stress Echocardiography Picano E, editor. 6th edition. 34 Chapter (pp. 551-568). New York: Springer-Verlag; 2015.

5. Olivotto I, Maurizi N. Exercise testing in hypertrophic cardiomyopathy: a pathophysiological goldmine with protean clinical implications. Editorial comment. Int J Cardiol 2019; 257-259.

6. Lancellotti P, Pellikka PA, Budts W, et al. Stress Echocardiography in Non-Ischaemic Heart Disease. J Am Soc Echocardiogr 2017; 30: 101-138. doi: 10.1016/j.echo.2016.10.016

7. Picano E, Ciampi Q, Wierzbowska-Drabik K, et al. The new clinical standard of integrated quadruple stress echocardiography with ABCD protocol. Cardiovasc Ultrasound 2018; 16: 1-12. doi: 10.1186/ s12947-018-0141-z

8. Cortigiani L, Carpeggiani C, Landi P, et al. Usefulness of Blunted Heart Rate Reserve as an Imaging-Independent Prognostic Predictor During Dipyridamole Stress Echocardiography. Am J Cardiol 2019; 124: 972-977. doi: 10.1016/j.amjcard.2019.06.017

9. Ciampi Q, Zagatina A, Cortigiani L, et al. on behalf of the Stress echo 2020 study group. Functional, anatomic and prognostic correlates of coronary flow velocity reserve during stress echocardiography. J Am Coll Cardiol 2019; 74: 2280-2293

10. Lazzeroni E, Picano E, Dodi C, et al. Dipyridamole echocardiography for diagnosis of coexistent coronary artery disease in hypertrophic cardiomyopathy. Am J Cardiol 1995; 75: 810-813. doi: 10.1016/s0002-9149(99)80417-2

11. Okeie K, Shimizu M, Yoshio H, et al. Left ventricular systolic dysfunction during exercise and dobutamine stress in patients with hypertrophic cardiomyopathy. J Am Coll Cardiol 2000; 36: 856-863. doi: 10.1016/S0735-1097(00)00818-4

12. Peteiro J, Bouzas-Mosquera A, Fernandez X, et al. Prognostic value of exercise echocardiography in patients with hypertrophic cardiomyopathy. J Am Soc Echocardiogr. 2012; 25: 182-189. doi: 10.1016/j.echo.2011.11.005

13. Peteiro J, Fernandez X, Bouzas-Mosquera A, et al. Exercise echocardiography and cardiac magnetic resonance imaging to predict outcome in patients with hypertrophic cardiomyopathy. Eur Heart J Cardiovasc Imaging 2015; 16:423-432. doi: 10.1093/ehjci/ jeu225

14. Ciampi Q, Olivotto I, Gardini C, et al. Prognostic role of stress echocardiography in hypertrophic cardiomyopathy: The International Stress Echo Registry. Int J Cardiol 2016; 219: 331-338. doi: 10.1016/j.ijcard.2016.06.044

15. Picano E, Scali MC, Ciampi Q, et al. Ultrasound for the Cardiologist. JACC Cardiovasc Imaging. 2018; 11: 1692-1705. doi: https:// doi.org/10.1016/j.jcmg.2018.06.023

16. Scali MC, Zagatina A, Ciampi Q, et al. The Functional Meaning of B-Profile During Stress Lung Ultrasound. JACC Cardiovasc Imaging 2019; 12: 928-930. doi: https://doi.org/10.1016/j.jcmg.2018.10.017

17. Wierzbowska-Drabik K, Picano E, Bossone $E$, et al. The feasibility and clinical implication of tricuspid regurgitant velocity and pulmonary flow acceleration time evaluation for pulmonary pressure 
assessment during exercise stress echocardiography. Eur Hear $\mathrm{J}$ Cardiovasc Imaging 2019; 20: 1027-1034. doi:10.1093/ehjci/jez029 18. Scali MC, Cortigiani L, Simionuc A et al. Exercise-induced B-lines identify worse functional and prognostic stage in heart failure patients with depressed left ventricular ejection fraction. Eur J Heart Fail. 2017; 19: 1468-1478. doi: 10.1002/ejhf.776

19. Gregor P, Čurila K. Medical treatment of hypertrophic cardiomyopathy - What do we know about it today? Cor Vasa 2015; 57 : e219-e224. doi: https://doi.org/10.1016/j.crvasa.2015.02.003

20. Bombardini T, Correia MJ, Cicerone C, et al. Force-frequency relationship in the echocardiography laboratory: a noninvasive assessment of Bowditch Treppe? J Am Soc Echocardiogr 2003; 16 : 646-655. doi: 10.1016/S0894-7317(03)00221-9

21. Cortigiani L, Huqi A, Ciampi Q, et al. Integration of Wall Motion, Coronary Flow Velocity, and Left Ventricular Contractile Reserve in a Single Test: Prognostic Value of Vasodilator Stress Echocardiography in Patients with Diabetes. J Am Soc Echocardiogr 2018; 31: 692-701. doi: 10.1016/j.echo.2017.11.019

22. Somura F, Izawa $H$, Iwase $M$, et al. Reduced myocardial sarcoplasmic reticulum $\mathrm{Ca}(2+)$-ATPase mRNA expression and biphasic force-frequency relations in patients with hypertrophic cardiomyopathy. Circulation 2001; 104(6): 658-663. doi:10.1161/hc3101.093869 23. Morimoto R, Okumura T, Bando YK, et al. Biphasic Force-Frequency Relation Predicts Primary Cardiac Events in Patients With Hypertrophic Cardiomyopathy. Circ J 2017; 81: 368-375. doi: 10.1253/ circj.CJ-16-1007

24. Green EM, Wakimoto $H$, Anderson RL, et al. A small-molecule inhibitor of sarcomere contractility suppresses hypertrophic cardiomyopathy in mice. Science 2016; 351: 617-621. doi: 10.1126/science.aad3456

25. Cecchi F, Olivotto I, Gistri R, et al. Coronary microvascular dysfunction and prognosis in hypertrophic cardiomyopathy. $\mathrm{N}$ Engl $\mathrm{J}$ Med 2003; 349: 1027-1035. doi: 10.1056/NEJMoa025050

26. Picano E, Pálinkás $A$, Amyot $R$. Diagnosis of myocardial ischemia in hypertensive patients. J Hypertens 2001; 19: 1177-1183. doi: 10.1097/00004872-200107000-00001

27. Youn H-J, Park C-S, Cho E-J, et al. Pattern of exercise-induced ST change is related to coronary flow reserve in patients with chest pain and normal coronary angiogram. Int J Cardiol 2005; 101: 299_ 304. doi: 10.1016/j.ijcard.2004.03.037

28. Camici P, Chiriatti G, Picano E et al. Noninvasive identification of limited coronary flow reserve in hypertrophyc cardiomyopathy. Cor Artery Disease 1992; 3: 513-521.

29. Lazzeroni E, Picano E, Morozzi L, et al. Dipyridamole-induced ischemia as a prognostic marker of future adverse cardiac events in adult patients with hypertrophic cardiomyopathy. Echo Persantine Italian Cooperative (EPIC) Study Group, Subproject Hypertrophic Cardiomyopathy. Circulation 1997; 96: 4268-4272. doi: 10.1161/01. cir.96.12.4268

30. Vasken D, Robert 0. Bonow, Stephen E. Epstein, et al. Myocardial Ischemia Detected by Thallium Scintigraphy Is Frequently Related to Cardiac Arrest and Syncope in Young Patients With Hypertrophic Cardiomyopathy. J Am Coll Cardiol 1993; 22: 796-804. 31. Tesic M, Djordjevic-Dikic A, Beleslin B, et al. Regional Difference of Microcirculation in Patients with Asymmetric Hypertrophic Cardi- omyopathy: Transthoracic Doppler Coronary Flow Velocity Reserve Analysis. J Am Soc Echocardiogr 2013; 26: 775-782. doi: 10.1016/j. echo.2013.03.023

32. Cortigiani L, Rigo F, Gherardi S, et al. Prognostic implications of coronary flow reserve on left anterior descending coronary artery in hypertrophic cardiomyopathy. Am J Cardiol 2008; 102: 1718-1723. doi: 10.1016/j.amjcard.2008.08.023

33. Nemes A, Balázs E, Soliman OI, et al. Long-term prognostic value of coronary flow velocity reserve in patients with hypertrophic cardiomyopathy: 9-year follow-up results from SZEGED study. Heart Vessels 2009; 24: 352-356. doi: 10.1007/s00380-008-1131-0 34. Sekine T, Daimon M, Hasegawa R, et al. Cibenzoline improves coronary flow velocity reserve in patients with hypertrophic obstructive cardiomyopathy. Heart Vessels 2006; 21: 350-355. doi: 10.1007/s00380-006-0917-1

35. Taqueti VR, Di Carli MF. Coronary Microvascular Disease Pathogenic Mechanisms and Therapeutic Options. J Am Coll Cardiol 2018; 72: 2625-2641. doi: 10.1016/j.jacc.2018.09.042

36. Brubaker PH, Kitzman DW. Chronotropic incompetence: causes, consequences, and management. Circulation. 2011; 123: 10101020. doi: 10.1161/CIRCULATIONAHA.110.940577

37. Magri D, Agostoni $P$, Sinagra G, et al. Clinical and prognostic impact of chronotropic incompetence in patients with hypertrophic cardiomyopathy. Int J Cardiol 2018; 271: 125-131. doi: 10.1016/j.ijcard.2018.04.019

38. Luo H-C, Dimaano VL, Kembro JM, et al. Exercise heart rates in patients with hypertrophic cardiomyopathy. Am J Cardiol 2015; 115: 1144-1150. doi:10.1016/j.amjcard.2015.01.548

39. Feneon D, Schnell F, Galli E, et al. Impact of exercise-induced mitral regurgitation on hypertrophic cardiomyopathy outcomes. Eur Heart J Cardiovasc Imaging 2016; 17: 1110-1117. doi:10.1093/ehjci/ jev242

40. Rowin EJ, Maron BJ, Olivotto I, et al. Role of Exercise Testing in Hypertrophic Cardiomyopathy. JACC Cardiovasc Imaging 2017; 10 : 1374-1386. doi: https://doi.org/10.1016/j.jcmg.2017.07.016

41. Maron BJ, Rowin EJ, Casey SA, et al. What Do Patients With Hypertrophic Cardiomyopathy Die from? Am J Cardiol 2016; 117: 434-435. doi: 10.1016/j.amjcard.2015.11.013

42. Picano E, Vañó E, Rehani MM, et al. The appropriate and justified use of medical radiation in cardiovascular imaging: a position document of the ESC Associations of Cardiovascular Imaging, 41. Percutaneous Cardiovascular Interventions and Electrophysiology. Eur Heart J 2014; 35: 665-672. doi:10.1093/eurheartj/eht394

43. Hirschfeld JW jr, Ferrari FA, Bengel FM, et al. 2018 ACC/HRS/ $\mathrm{NASCI} / \mathrm{SCAI} / \mathrm{SCCT}$ Expert consensus document on optimal use of ionizing radiation in cardiovascular imaging :best practices for safety and effectiveness. JACC 2018; 24: e286-e348.

44. Young JR, Orosz I, Franke MA, et al. Gadolinium deposition in the paediatric brain: T1-weighted hyperintensity within the dentate nucleus following repeated gadolinium-based contrast agent Clin Radiol 2018; 73: 290-295. doi: 10.1016/j.crad.2017.11.005.

45. Maron BJ, Maron MS, Maron BA, et al. Moving Beyond the Sarcomere to Explain Heterogeneity in Hypertrophic Cardiomyopathy. J Am Coll Cardiol 2019; 73: 1978-1986. doi:10.1016/j. jacc.2019.01.061 